\title{
3 Research Square

\section{In situ determination of the extreme damage resistance behavior in stomatopod dactyl club}

\section{Zheng Dong}

Institute of High Energy Physics

\section{S. Chen}

Southwest Jiaotong University

\section{Himadri Gupta}

Queen Mary University of London

\section{Xiaoyi Zhao}

Institute of High Energy Physics

Yiming Yang

Institute of High Energy Physics

\section{Guangcai Chang}

Institute of High Energy Physics

Jian Xue

Institute of Mechanics

\section{Yiyang Zhang}

Southwest Jiaotong University

S. N. Luo

Key Laboratory of Advanced Technologies of Materials, Ministry of Education, Southwest Jiaotong University

Yuhui Dong

Chinese Academy of Sciences https://orcid.org/0000-0002-4422-4685

Yi Zhang ( $\square$ zhangyi88@ihep.ac.cn )

Institute of High Energy Physics

\section{Article}

Keywords: stomatopod dactyl, in situ characterization, 3D crack evolution, fiber bridging, toughening mechanisms

Posted Date: July 20th, 2021

DOI: https://doi.org/10.21203/rs.3.rs-698848/v1 
License: (c) (i) This work is licensed under a Creative Commons Attribution 4.0 International License. Read Full License

Version of Record: A version of this preprint was published at Journal of Synchrotron Radiation on March 14th, 2022. See the published version at https://doi.org/10.1107/S1600577522001217. 


\section{Abstract}

The structure and mechanical properties of the stomatopod dactyl club have been extensively studied for its extreme impact tolerance, but a systematic, in situ, investigation on the multiscale mechanical responses under high-speed impact has not been reported. Here we reveal the full dynamic deformation and crack evolution process within projectile impacted dactyl using combined fast $2 \mathrm{D}$ x-ray imaging and high resolution ex-situ tomography We show hydration states can result in significantly different toughening mechanisms inside dactyl under dynamic loading. We find a previously unreported 3D interlocking structural design in the impact surface and impact region using nano x-ray tomography (nano-CT). Experimental results and dynamic finite element modeling (DFEM) suggest this unique structure plays an important role in resisting catastrophic structure damage and hindering crack propagation. Our work will help to understand the key toughening strategies of biological materials and provide valuable information for biomimetic manufacturing of impact resistant materials in general.

\section{Introduction}

Lightweight, high-strength and high-toughness composite materials are continually developed and improved to meet ever-increasing performance needs in construction, aerospace, and defense sectors ${ }^{1,2}$. However, a better balance between strength and toughness is still challenging to achieve in traditional material manufacturing and deemed impractical in producing lightweight materials ${ }^{3}$. To address this challenge and develop materials with an excellent combination of strength and toughness under highspeed impact, scientists and engineers seek inspirations from natural materials ${ }^{4-8}$, such as scales ${ }^{9}$, nacre ${ }^{10}$, and antlers ${ }^{11}$. In comparison with engineering materials, natural materials tend to achieve a better balance between strength and toughness, through excellent structural design across multiple length scales, from the molecular to the macroscopic ${ }^{3,4}$.

The stomatopod dactyl club (Fig. 1b), which acts as the offensive organ during preying and ritualized fighting, is one of the toughest and most impact resistant biological tissues in nature ${ }^{12,13}$. The exoskeletons of stomatopod dactyl exhibit remarkable tolerance against dynamic impacts which can induce catastrophic damage on the shells of prey like crab, mollusk etc ${ }^{14}$. As these creatures use the same environment to obtain the elements making up their exoskeleton, the stomatopod must have adapted superior structure optimization strategies to develop its dactyl clubs compared to its prey. It is therefore an ideal natural material for research in optimizing strength and toughness for high-speed impact-resistant biomimetic composites.

Extensive studies have been performed in the past ten years to uncover the structural optimization strategies and toughening mechanisms of the stomatopod dactyl, generating numerous characterization results, and various intrinsic and extrinsic toughening mechanisms have been derived ${ }^{15-21}$. To date, these studies already provided innovative inspirations for the manufacture of high-energy absorption and impact-resistant composite materials in industry ${ }^{22-24}$. One of the first comprehensive reports on 
stomatopod cuticles suggested they are made of three distinct regions: the impact region, the periodic region, and the striated region ${ }^{15}$ (Fig. 1c). X-ray diffraction (XRD), energy-dispersive spectroscopy (EDS), and nanoindentation combined with dynamic finite element analysis (DFEA) were performed in this study, leading to several important conclusions, including that the origin of the internal cracks is located near the impact surface (the periodic region), and that the oscillating elastic modulus of adjacent lamellae as well as a larger-scale modulus mismatch between different regions plays important roles in preventing and deflecting cracks. A second study ${ }^{16}$ demonstrated that the chemical and microstructural variation of the mineral distribution in the tissue periphery strengthens the dactyl. Nanoindentation tests with a micrometer spatial resolution revealed graded, quasi-plastic mechanical responses in the outer region of the dactyl, including misalignment of hydroxyapatite (HAP) crystallites (sliding and rotation), and strain hardening, attributed to chitin and amorphous mineral interactions, resulting in significant energy dissipation by localized yielding before shear-induced circumferential cracks are nucleated ${ }^{17,20}$. Recently, a further experiment-based quantitative analysis demonstrated plastic dissipation at the crack tip is the main contribution to the fracture resistance, with both linear elastic fracture mechanics (LEFM) and elastic-plastic fracture mechanics (EPFM) protocols tested. By using nanoindentation to initiate cracks, it was also found that the cracks can be deflected by out-of-plane fibers ${ }^{18}$. Finite element analysis and simulation tests using biomimetic 3D printed structures suggested that the herringbone architecture in the interface of the impact surface and impact region of dactyl may offers an enhancement in stress redistribution and out-of-plane stiffness in responding to compressive loading on top of helicoidally structured dactyl cuticle ${ }^{18}$. Another nanoindentation study with high-strain rates (around $10^{4} \mathrm{~s}^{-1}$ ) nanoindentation demonstrated that the toughness of its hard mineral surface consisting of highly regularly arranged nanoparticles was related to impact strain rate. The mineral coating has exceptional energy dissipation efficiency under high-strain-rate impact, and is able to localize damage and prevent crack initiation and propagation under high-speed impact ${ }^{20}$.

However, the aforementioned structure properties and toughening mechanisms were mainly uncovered by static or quasi-static structural and mechanical characterizations. Most of the studies are restricted to investigating the local structure and mechanical properties of stomatopod dactyl cuticle, as opposed to in situ dynamic visualization, due to the limitation of the applied characterization methods. The structuralmechanical mechanisms across hierarchical levels under dynamic loading are relatively few and remain to be explored so far. In fact, systematic investigation and analysis on multiscale material properties of stomatopod dactyl still heavily rely on modelling e.g., finite element method (FEM) and other simulation approaches. The lack of experimentally verified structure and material parameters as inputs at the extreme strain-rates and loads involved, undermines the credibility of the simulation results. The loading strain rate is a crucial factor that can affect the mechanical behaviors, and therefore, experimental characterization under realistically high impact rates (around $10^{6} \mathrm{~s}^{-1}$ ) is crucial to uncover the dynamic structure-property relations of stomatopod dactyls. However, there are still no multiscale in situ structural and dynamic mechanical studies reported till now. 
To study the multiscale dynamic deformation mechanisms of dactyl club is technically challenging. Ideally a method with a high 3D spatial resolution (micrometer scale) and a large field of view is needed to examine the hierarchically structured dactyl, and the measurement has to be fast (microsecond level resolution). Synchrotron $\mathrm{x}$-ray imaging and tomography methods are powerful and nondestructive tools enabling the probing of $2 \mathrm{D}$ and $3 \mathrm{D}$ structural properties and deformation behavior of materials ${ }^{25}$. X-ray transmission measurements can potentially determine micrometer-level strains on a microsecond temporal-scale. On the other hand, it is highly desirable to study the mechanical behavior of the heterogeneously structured dactyl club without the ability to assess the deformation on a 3D volumetric basis. While conventional micro-CT can provide static 3D structure characterizations of materials from the micro- to meso-scales and in some cases $4 \mathrm{D}$ X-ray micro-CT can be used to examine the internal deformation behavior and fracture processes in different loading situations ${ }^{26}$. However, micro-CT techniques are generally not applicable for fast characterization of structures ranging from multiple length scales due to a contradiction of spatial and temporal resolutions with a desired field of view. So far, the spatial and temporal resolutions of synchrotron X-ray micro-CT techniques are far from capable to view the damage and facture process occurring inside the whole dactyl club. Hence, a customized characterization strategy taking advantage of the merits of different methods was employed for this project.

Herein, we performed in situ spherical projectile penetration tests (Fig. 1d) on the stomatopod dactyl using an in-house system developed to mimic high-strain rate $\left(\sim 10^{6} \mathrm{~s}^{-1}\right)$ impact loading situations encountered by the stomatopods in nature. A combination of real-time 2D x-ray imaging and highresolution postmortem tomography examination was employed to investigate the structure evolution induced by dynamic loading of the dactyl club. Equipped with a superconducting wiggler, the 3W1 beamline of Beijing Synchrotron Radiation Facility (BSRF) offers fast x-ray imaging capability to perform in situ characterization of the nucleation and propagation of cracks in 2D, while high resolution postmortem micro-CT was used to examine the 3D internal structure in detail. We revealed the full crack evolutionary process and other previously unreported toughening mechanisms inside the dactyl club, and substantial differences in mechanical responses caused by hydration variation. Furthermore, a detailed structural characterization on the impact surface and impact region using nano-CT, combined with an unprecedented dynamic finite element modeling (DFEM) simulation based on the reconstructed structure, was conducted to unveil the unknown mechanical roles of pore canal networks, chitin fiber scaffolds and mineral particles within this critical region of the dactyl club.

\section{Results}

Nucleation sites and crack deflection. High-speed synchrotron x-ray 2D imaging with a super-short interval of $5 \mu \mathrm{s}$, exposure time of $6 \mu \mathrm{s}$ and spatial resolution of $4 \mu \mathrm{m}$ was employed to visualize the initiation and propagation of cracks in the stomatopod dactyl cuticle during the in situ impacting process (Fig. 2a-f). As shown in the representative images, the crack nucleation and propagation processes within the dactyl were captured while the sample was impacted by a spherical projectile with a diameter of 500 
$\mu \mathrm{m}$ at speed of $160 \mathrm{~m} / \mathrm{s}$. The white strips emerged in the x-ray images featured the cracks induced by the impacting energy. As the cracks transmitted along the impacting direction, clear in-plane (indicated by the horizontal white strips) deflections toward impact surface were initiated.

The results show significant local fracture was mainly constrained within the impact region beneath the projectile contacting point with a length-scale about $160 \mu \mathrm{m}$. As the impacting process evolves, various damage mechanisms were observed, including sequential cleavage fracture (Fig. 2b), wing (or secondary) crack nucleation (Fig. 2c), crack deflection and coalescence (Fig. 2e) ${ }^{27}$. Even though the contrast of the acquired 2D images is not ideal, they provide valuable in situ information on the occurrence and evolution of different crack systems, of great importance for tracing and analyzing the 3D structure information from 3D ex-situ tomography experiments. To uncover the entire crack paths induced by the projectile impacting power, an ex-situ x-ray tomography imaging was performed on the same sample. As shown in Fig. $2 \mathrm{l}$ and $2 \mathrm{~m}$ the full 3D crack systems induced by the impacting were successfully captured. The projection image with pixel size of $3.25 \mu \mathrm{m} \times 3.25 \mu \mathrm{m}$ collected from the same perspective (Fig. 2g) as in the in situ 2D imaging offer clearer view of the crack paths within the impact and periodic region. The cracks which were propagating in the different lamellae shown low coalescence (Fig. 2h, 2k and 2m), along with branching (Fig. 2j) and deflecting behavior and were guided towards the dactyl surface in the end (Fig. $2 \mathrm{~h}$ and $2 \mathrm{~m}$ ). Four major crack deflection sites were identified and indicated by arrows in different colors. Figure $2 \mathrm{i}-\mathrm{k}$ show the sliced images of the 3D reconstruction along the dashed lines in the Fig. $2 \mathrm{~g}$. The multiple nucleation sites and growth of crack with low coalescence may lead to stress alleviation, which would be beneficial for the integrity of the dactyl club ${ }^{28}$. The four major deflection sites can be easily spotted in the CT slice (Fig. 2i, along the orange dash line) image, the first major deflection (green arrow) occurred before the main crack propagated to the impactperiodic interface. This phenomenon demonstrated the remaining impacting energy was not sufficient to penetrating the fronting lamella, therefore inclined to spread between lamellae looking for weak point to conquer. The crack path between the yellow and orange arrow shows that the crack travelled a long distance along the impact-periodic interface, indicating the interface between two major regions is more favorable for crack spreading compare to the interface between two lamellae within the same region (detail information in Supplementary Fig. 1 and Supplementary Note 1). The result further verified the larger modulus mismatch between impact and periodic regions (with Dundurs' parameter of 0.33 at this interface) is important to hinder crack propagation through-thickness ${ }^{15,18}$. The crack path between the orange and red arrow exhibits a zigzag shape within the periodic region suggesting the deflection occurred more frequently than in impact region, this may be due to the loosely packed structure and larger pore canals in this region. The frequent crack deflection significantly delayed catastrophic fracture and increased in crack surface area, leading to improved fracture resistance ${ }^{29}$.

As shown in Fig. $2 \mathrm{~h}$ and $2 \mathrm{k}$, the crack was quickly deflected back into the impact region and finally reach the dactyl surface where the remaining energy was fully dissipated. The CT slice image (Fig. 2j, along the yellow dash line) shown that crack branching was induced in the first major deflection site. In the CT slice further away from the impacting point, the main crack turned into multiple microcrack networks, and the 
crack path shown in Fig. $2 \mathrm{k}$ and $2 \mathrm{~m}$ appear to be discontinuous. The zigzag shaped crack propagation path can be seen more clearly from the high magnification SEM image (Fig. 3c and Supplementary Fig. 2a) collected on the cross section of the impacted dactyl. Figure 3c-i shows how the crack penetrated through a lamella when it has enough energy, with the high stress likely tearing the fiber sheets to propagate across different lamellar layers. Separation of adjacent lamellae was also found as shown in Fig. $3 \mathrm{c}$ and Fig. 3c-ii. Figure 3c-ii provides a rare view of how the crack is propagated within the weak interface of two neighboring lamellae, where the crack path was nearly at its end, with the remaining energy consumed by the fracture of fiber bundles (Supplementary Fig. 2b). As known previously ${ }^{15}$, in the periodic region, the fiber bundles are arranged following the shape of dactyl clubs, while the arrangement undergoes an abrupt change in the periphery of striated region, leading to abrupt crack propagation direction changes correspondingly. The complex 3D route of crack propagation results in a significant increase in fracture toughness, and demonstrated the overall energy absorption can be affected by the macroscale morphological characteristics ${ }^{4}$.

Although equipped with a superconducting wiggler, the $3 \mathrm{~W} 1$ beamline is based on a first-generation synchrotron source, the lack of coherence and low flux of x-ray prevent us from obtaining clearer and shorter time-interval images. For hydrated samples, the impact energy of incident projectile is not high enough to initiate visible cracks that can be observed by our in situ 2D x-ray imaging equipment. However, the hydration states of the dactyl sample are expected to play an important role in conducting its dynamic mechanical behaviors ${ }^{15,17}$. Ex-situ $x$-ray tomography and SEM measurements were employed to investigate the toughening and energy dissipation mechanisms of the dactyl club under the same loading conditions. According to the 3D crack networks, the volume of the main crack systems generated within the sample is about 20 times smaller than dry sample under similar impact energy. The results indicated that for the dry samples more energy was dissipated by brittle fracture such as crack initiation and extension, while for wet sample more energy tend to be consumed by other toughness mechanisms like plastic deformation and fiber bridging etc. As shown in Fig. 3d, several crack systems were nucleated at multiple sites away from the impact point, while surprisingly no main crack was found in the site directly beneath impact point, which means that there might have a zone of plastic deformation beneath the impact point.

Unlike the cracks deflected around the interface of impact-periodic region in dry sample, the cracks within hydrated samples deflected at the interface of impact surface/impact region (Denoted in Fig. 3d with black arrow). Slices of reconstructed volume for hydrated sample (Fig. 3e-f) show the main crack deflected in impact region and interface of impact surface-impact region. Distinct from the main cracks of the dry sample, the main cracks of the hydrated sample are discontinuous, as denoted in Fig. $3 \mathrm{~d}$ with blue arrow. The discontinuity of crack system in the wet sample may is mainly caused by the herringbone architecture in the impact region which was identified as a crucial structure feature for enhancing energy dissipation in literature ${ }^{15}$. The complex fiber orientation distribution within the herringbone structure may induce abrupt disruption against crack propagation and therefore reduce the integrity of the main crack system. The impact energy were dissipated more efficiently in the impact surface for wet sample, hence 
the remaining energy was difficult to penetrate the herringbone structure compare to the dry sample. Another toughening mechanism observed in dactyl (which is hydration sensitive) is plasticity at the nanoscale. From SEM images of cross section of damaged hydrated dactyl club (Fig. $3 \mathrm{~g}$ ), there is a distinct plastic zone (Fig. 3g-ii) beneath the impact point, where highly misaligned mineral crystallites can be observed. The reorientation behaviors of the mineral crystallites were previously found in the impact surface of dactyl club when under high strain rate nanoindentation loadings ${ }^{17}$, resulting from shear band localization of the stress waves. Figure 3g-iii shows an elastic zone featuring with textured arrangement of mineral crystallites. At the impact point, visualized at high-magnification in Fig. 3g-i, the existing of large pores might result from the loss of broken mineral particles. Under high stress, the crystallites fixed on the fibers were smashed into small irregular particles ${ }^{20}$ and exfoliated during polishing, leading to pores. We also observed fibers bridging the tips of opened microcracks (Fig. 3g-iv) within the elastic zone and fracture of fiber bundles (Fig. 3g-v). Under high-speed the impact, beneath the impact point the primary toughening mechanism is plasticity, which involves material phenomena like particle breakage, rotation, translation as well as microcracks, rather than crack deflection.

Deformation behavior of mineral nanoparticles. The impact surface (about $70 \mu \mathrm{m}$ in thickness ${ }^{18}$ at the outermost region of the dactyl) which mainly consists of FAP nanoparticles ${ }^{16}$ (about $60 \mathrm{~nm}$ in diameter ${ }^{20}$ ) was considered to be mechanically crucial for providing the first shield against impact in recent studies ${ }^{20}$. Various toughening mechanisms have been found in this region from previous studies, such as: reorientation of crystallites, particle breakage/rotation/translation ${ }^{17,20}$. However, several new forms of deformation behavior have been revealed by investigating the damaged surface through SEM.

Figure 4a and Fig. 4b show the post-impact images of the damaged surface on the dry and hydrated dactyl club, where catastrophic fracture and spalling occurred on the impact surface when subjected to high-speed loading $(160 \mathrm{~m} / \mathrm{s})$. As mentioned before, the impact induced a plastic zone surrounding the loading point on the hydrated sample. The main fracture damage was restrained in a limited region with a diameter of $700 \mu \mathrm{m}$ (Red circle in Fig. 4c with radius labeled by $r_{2}$ ). For convenience, we labeled the surface area with R1 (within $r_{1}$ ) and R2 (between $r_{1}$ and $r_{2}$ ) region according to different radius from the central impacting point. Close to the loading point where largest impact forces were applied (R1; an area with a diameter of about $70 \mu \mathrm{m}$ ), misalignment of mineral nanoparticles can be observed within this region (Fig. $4 \mathrm{f}$ within region R1 comparison with Fig. 4g within region R2). Away from the central zone, where the impact forces were not sufficient to break the nanoparticles, particle rotation and translation seems to be the main means to consume the remaining energy within R2 (Fig. 4j within region R2 comparison with Fig. $4 \mathrm{k}$ within the outer region). Also, the compression of the fiber layers and the nanoparticles also contribute to the energy absorption (Fig. 4h shows the compressed fiber layers while Fig. $4 \mathrm{i}$ shows the uncompressed). As the damage area extended further, the spalling path was redirected towards the surface at the edge of the circle ( $\mathrm{r} 2$ ). The CT images (Fig. 3e and 3f) show that the deflection of the spalling occurred at the depth about $100 \mu \mathrm{m}$ when it reached the interface of the impact surface and impact region. The observed phenomenon further verified the hypothesis that the unique interlocking interfaces of the two regions can enhance stress redistribution and out-of-plane stiffness, acting as an 
important shield to prevent the catastrophic fracture going deeper. Under a higher speed $(200 \mathrm{~m} / \mathrm{s})$ impacting, the diameter of damage region has no significant change, but severe fracture took place at impact point, as shown in Fig. 4b.

Multiscale fiber bridging behavior. Fiber bridging can effectively reduce the local stresses and strain fields at the crack tip and acts as a prevailing toughening mechanism of the hierarchical biocomposite materials. ${ }^{3}$ The association of fiber bridging with crack evolution and energy absorption inside biocomposites has been systematically studied in many literatures ${ }^{30-33}$. Here, many new forms of fiber bridging behavior were captured within the impacted dactyl club.

A wider range of bridge length (tens of $\mathrm{nm}$ to $\mu \mathrm{m}$, shown in Fig. $4 \mathrm{e}$ and Fig. 5 respectively) was examined inside the samples that suffered fast impact compared to those (on the order of $\mu \mathrm{m}$ ) subjected to quasistatic loading 17,18 , indicating that the energy dissipation through crack bridging cannot be ignored under high-speed impact. As is known, the chitin fibrils ( 3-4 nm in diameter) self-assemble with protein to form nanofibers ( 20-100 nm in diameter). At the next hierarchical level, nanofibers are partially mineralized with calcium carbonate to form planar arrays of fibers (fiber sheets) stacked in a herringbone style structured manner to form lamellae at microscale within impact region ${ }^{34}$. The multiscale fiber pulling-out and bridging scheme are shown in Fig. 5d (Fiber bridging away from the impact point) and Fig. $5 e$ (Fiber bridging near the impact point) which offers valuable information to deduce the related energy dissipation behavior: fiber sheets pulled from fiber lamellae (Fig. 5b, Fig. 5d-i and 5d-ii), fiber bundles pulled out from fiber sheets (Fig. $5 \mathrm{~d}$-iv and Fig. 5e-iii), fibers pulled out from fiber bundles (Fig. 5e-iii and 5e-iv) and nanofibers pull out from fibers (Fig. 5d-iv and Fig. 5e-v). The frictional energy for pulling out fibers at different scale could all contribute to the toughening. Pulling out of fiber bundles from the unique herringbone style structured interface will be much harder as different fiber layers will be involved and easily result in large scale twisted fiber bridging (Fig. 5b). Also, the out-of-plane fiber bundles running through the pore canals will exert extra resistance. Further, the pulling out process of the in-plane fibers will cause the contraction of the pore canals (Fig. 5d-ii) and forming isolated fiber sheets, along with fracture behavior of the out-of-plane fibers (Fig. 5d-iii). At the sites where fiber bridging is initiated, the nanofibers still remain connected while the surrounding matrix is fractured and full of visible nanocracks (Fig. 5d-iv), demonstrating a significant nanoscale toughening mechanism such as strain energy stored in the elastic fibers and the debonding energy for the fracture of mineral aggregation ${ }^{35}$. Remarkably, high magnification SEM images revealed how fiber bridging form in the lower hierarchical level and its association with the energy absorption. Figure $5 e(i-v)$ shows a remarkable fractal fiber bridging behavior near the impact point across multiple structural level. The bridges connecting lamellae are large fiber bundles (tens to hundreds of micrometers, Fig. 5e-ii and Fig. 5d-i); at the end of the bridges, the fiber bundles split into single fibers or smaller fiber bundles (several micrometers, Fig. 5e-iii); further nanofibers inside those fibers are released from the encasing matrix to form the nanoscale links (Fig. 5eiv). 
3D structural characterization of impact surface and impact region. As mentioned before, the interface and junction region between the different regions of dactyl clubs can act as crack deflectors, especially when the dactyl is under hydration state. The complex geometry of the interfaces and enormous elastic modulus mismatch between the impact surface and the impact region might be key structural feature for enhance energy dissipation and crack deflection ${ }^{15}$. However, the detailed 3D structures of this region are still relatively unknown.

To gain further understanding of this region, nano-CT was used to examine the structure properties in fine details (with 3D spatial resolution of $64 \mathrm{~nm}$ ). The reconstructive 3D volume shows that the outermost layer of the dactyl club was coated with mineral nanoparticles and no pore canals were observed (Fig. 6ad). Beneath, densely packed mineralized chitin fibers (Fig. 6a) were distributed across the impact region forming an interpenetrating pore canal network (Fig. 6b). Surprisingly, besides the out-of-plane pore canals orient normal to the surface of the dactyl club which were found in most regions of the cuticle by $\mathrm{SEM}^{18,19}$, interconnecting in-plane pore canals framework were exposed in impact region which bundle the vertical canals together. In addition, clear evidence can be seen in the 2D slices (Fig. 6c-d) of the CT images that the pore canals were covered by mineral nanoparticle coating across all regions. As the 3D pore canal network is speculated to play an important mechanical role other than serving as channels for material transport during molting ${ }^{17,36}$, the nano-CT results furnish a new perspective how the pore canals are mechanically strengthened. The nanoparticle coatings could provide further protection of the pore canal networks from catastrophic collapse by dissipating impact energy through possible toughening mechanisms, such as particle rotation, translation and microcracks ${ }^{15,17,20}$. And also, the distribution of mineral particles in different layers surrounding the pore canal networks can also act as crack deflectors resulting from the elastic modulus mismatch of hard-soft interface ${ }^{37}$, thus increasing the fracture toughness of dactyl club. Due to the elastic modulus mismatch, cracks could be redirected and propagate perpendicular to the original direction, which has been summarized as a Cook-Gordan crack-stopping mechanism ${ }^{38}$. The strong correlation between the morphology of the mineral coating distribution and the pore canal networks as shown in Fig. 2e-i, ii and iii also suggests that the mineralization process might be highly dependent on mineral ion supply from the pore canal systems. Moreover, a thin section of segmented 3D volume of mineral particles and fiber bundles (Fig. 6e-iv) indicates irregular suture interface design of impact surface and impact region. These images show that at the interface, the fiber bundles within impact region are inserted into the mineral coating within impact surface. Geometrically interlocking interfaces enhance interfacial stiffness and strength, and interface waviness increases resistance to crack propagation ${ }^{39}$. SEM images also illustrates the insertion of fiber bundles and mineral coating (Supplementary Fig. 3e).

Dynamic finite element analysis (DFEA) was performed to gain a further insight on the mechanical role of newly uncovered structures in the impact surface and impact region. The geometrical shapes and dimensions used in the analytical model was based on the 3D architectural structure (Fig. 7a) acquired from nano-CT test. Figure 7b shows a geometric model of dactyl tissue which was converted to 3D finite element with high fidelity. To decouple the mechanical effects between the complex nanoscale 
geometrical feature and elastic modulus mismatch in the interface area, two specific models were defined with different material property assignments. For model I, different elastic modulus properties were assigned to the impact surface and impact region using values reported in literature ${ }^{15}$, therefore a high modulus mismatch ( $E_{1}=70 \mathrm{GPa}$ for mineral coating and $E_{2}=35 \mathrm{GPa}$ for fiber scaffold) exists at the interface. In contrast, for model II the elastic modulus properties of mineral and fiber in the region were set to be the same on purpose, to focus on analyzing the geometrical effects on the mechanical performance.

The impact target was set to be a rigid ball with radius of $1 \times 10^{-6} \mathrm{~m}$ and mass of $1 \times 10^{-16} \mathrm{~kg}$ for both models. When subjected to impacts at a velocity of $200 \mathrm{~m} / \mathrm{s}$, the average Von-mises stress distribution cloud diagrams of model I and model II are shown in Fig. 7c and 7d, respectively. At the initial stage (first $0.5 \mathrm{~ns}$ ), the Von-mises stress in both models were still distributed in the surrounding areas of the contact point. A peak stress $(\sim 1150 \mathrm{MPa})$ was obtained near the impact contact zone at $0.25 \mathrm{~ns}$ and the value deceased significantly ( $300 \mathrm{MPa})$ in the next $0.25 \mathrm{~ns}$. Notably, the initial semi-spherical shape stress pattern indicating a continuous propagation was disrupted in model I right after the compressive wave reached the interface of the impact surface and impact region at $0.75 \mathrm{~ns}$, while no clear disruption was shown in model II. The results demonstrate the modulus mismatch in the interface acts as the first shield to hinder the impact stress propagation. As the compressive wave travelled further into the interface region, and the complex interlocking structure of the mineral coating impact surface and bulk of the impact region came into effect, the spherical shape of the stress pattern starts to exhibit obvious deformation in both models, which suggests the interlocking geometry in the interface region plays a dominant role in shielding impact waves. As the compressive wave travels deeper into the dactyl club, clear stress concentration behaviors are found around pore canal regions in model I, while no visible stress concentration signs are shown in model II (1.25-1.5 ns). The results indicated the modulus mismatch at the pore canal region is the main reason for stress concentration. The hypothesis can be further proved by the 3D mineralized pore canal networks in Fig. 6e. The modulus mismatch hinders the stress and crack propagating from the mineral coating layers into the fiber scaffolds of the pore canals, leading to the deformation and reorientation of the mineral nanoparticles and nucleation of microcracks in the mineral layer. Considering the wide distribution and large area of pore canals (specific surface area about $7.73 \times 10^{5} \mathrm{~m}^{2} / \mathrm{m}^{3}$ ) within the impact region of dactyl club, the mineral particles in the pore canal networks would dissipate considerable energy during impact event. The mineral particles surrounding pore canal networks helps spread the damage to macroscopic tissue regions from a microscale initiation point that improving the toughness of dactyl club. This kind of mineral strengthening strategy might inspire the designs of biomedical implant materials which requires high mechanical strength and microscale porosity for nutrient flow and cell proliferation ${ }^{40}$. The FEMA results demonstrated that the elastic modulus mismatches at different length scales and the irregular suture interface of junction region can work together to prevent the stress from propagating into the bulk of impact region.

\section{Conclusion}


The stomatopod dactyl cuticle is a prime example of how structural and chemical arrangement of basic biological components can create tough, impact-resistant armor to withstand high dynamic external loadings. Its exquisite hierarchical structure endows the dactyl cuticle with different energy dissipation mechanisms. Through our in situ x-ray imaging measurements during a projectile penetration test which mimics the real impact situations encountered by the dactyl clubs, we experimentally observed the full process of crack initiation and evolution for the first time. Supplemented by CT and SEM results, a full 3D crack system which operates across multiple length scales was uncovered in great detail, as well as other toughness behaviors like formation of plastic regime and fiber bridging etc. The real time 2D images provided critical clues in determining the time sequence of the formation and evolution of large crack systems inside the dactyl club.

The crack initiation and development mechanisms found in the in situ impact mechanical test experimentally verified the simulation results and hypothesis reported previouly ${ }^{15}$. The imaging and CT results show clear evidence that the crack tends to initiate at the interfaces of different regions where a high modulus mismatch exists. The cracks have a tendency of deflection at the interface when propagating across different lamellae and regions. Furthermore, the influence of hydration states on the mechanical behavior of dactyl was investigated. The dry and hydrated samples exhibit different mechanical responses under the same loading energy, and the sites of crack nucleation and the volume of the crack systems vary significantly. In addition, other toughening mechanisms including formation of a plastic zone under the loading point, and multiscale fiber bridging systems, are unique for the hydrated samples. Moreover, a large extent of particle breakage and compaction was found beneath the contact region, which has not been observed in the low strain-rate nanoindentation tests ${ }^{17,20}$.

A combination of nano-CT examination and DFEM study was carried out for further understanding of the mechanical role of the impact surface and impact region. A previously unknown heavily interlocking structure formed by multiple components in the impact surface was revealed. The mineral coating in the impact surface region and the fiber bundles within impact region form an irregular suture like a transition zone with large tip angles ${ }^{41}$, leading to a larger area of hard-soft interface, hence more prone to experience ductile failure and inhibit catastrophic crack propagation. Another previously undiscovered structural feature was the unique network of interpenetrating pore canals in the impact region. A dense distribution of in-plane pore canals was found hiding in the impact region which bind the out-of-plane pore canals tightly, forming a firm interlocking system. The results also show that the 3D distributed pore canals were stiffened by mineral particles, which would more easily promote multiple initiation sites for the formation of more microcracks.

Our results demonstrated various toughening mechanisms over a wide range of length scales that, working together, contribute to the overall dynamic mechanical properties of stomatopod dactyl clubs. Such results provide key inspirations and design guidelines for the fabrication of next-generation impactresistant composite materials.

\section{Materials And Methods}


Live specimens of mantis shrimps (Odontodactylus scyllarus) were obtained from online retailers in Taobao and stored frozen at $-80^{\circ} \mathrm{C}$ till use. The dactyl clubs with a smooth surface were selected for in situ impact experiment. The dry dactyl used in the impact experiment were dried in a hot air oven at $30^{\circ} \mathrm{C}$ for $6 \mathrm{~h}$. The dactyl clubs after impacted were carefully stored in refrigerator before x-ray CT examination. Finally, for the SEM tests, the same samples were embedded in epoxy (SPI-Pon812) and then sectioned using a linear precision diamond saw IsoMet 4000 (Buehler), ground and polished sequentially with P1200/4000/5000/7500-grade SiC paper and finally polished with $1 \mu \mathrm{m}$ diamond paste, followed with a 10 min ultrasonic bath to remove debris from the sample surfaces ${ }^{17}$.

In situ dynamic imaging during impact. Impact experiments with in situ dynamic imaging on the dactyl club were carried out at the beamline 3W1 of BSRF where an in-house setup was developed for impact penetration with in situ phase contrast imaging $(\mathrm{PCl})$. High flux white x-ray beam generated by a superconducting wiggler was used to illuminate the dactyl sample and a high-speed x-ray imaging system was used for fast structure characterization of the dactyl clubs. As shown in the schematic diagram of the impact experiment setup (Fig. 1d), a dactyl sample was fixed at the sample stage waiting for the impact from an accelerated projectile with a diameter of $500 \mu \mathrm{m}$ at a speed ranging from 110 to $200 \mathrm{~m} / \mathrm{s}$. During impact, the frame rate of the $\mathrm{x}$-ray camera is set at 200,000 frames $\mathrm{s}^{-1}$, and the exposure time at $6 \mu \mathrm{s}$. The effective pixel size of the acquired image is about $4 \mu \mathrm{m} \times 4 \mu \mathrm{m}$.

X-ray Computed Tomography. To elucidate the cracks distribution, 3D x-ray CT characterizations were performed on the same damaged samples at the X-ray Imaging and Biomedical Application Beamline (BL13W1) in Shanghai Synchrotron Radiation Facility (SSRF). The nominal pixel size of the reconstructed image is about $3.25 \mu \mathrm{m} \times 3.25 \mu \mathrm{m}$. The $\mathrm{x}$-ray energy was set at $30 \mathrm{keV}$ and reconstructed into 3D images with software PITRE ${ }^{42}$. Subsequently, crack segmentation and analysis were conducted using the Avizo software.

Nano-CT characterization on the impact surface and impact region was carried out using a full-field transmission hard $\mathrm{x}$-ray microscopy system installed at the beamline 4W1A in BSRF with a beam energy of $8 \mathrm{keV}$. Finally, the volume spatial resolution is about $64 \mathrm{~nm}$. Segmentation and model meshing for DFEA were conducted with software Avizo.

Dynamic Finite Element Analysis (DFEA). Dynamic Finite Element Analysis (DFEA) of an impact event between the junction region of impact surface and impact region with a rigid target was designed using Avizo and analyzed using the commercial finite element software Abaqus/Explicit. The mechanical response of the material of model I was modeled as isotropic linear elastic with different mechanical properties $\left(E_{1}=70 \mathrm{GPa}\right.$ for mineral coating and $E_{2}=35 \mathrm{GPa}$ for fiber scaffold) and mass density $\left(\rho_{1}=\right.$ $3160 \mathrm{~kg} / \mathrm{m}^{3}$ for mineral coating and $\rho_{2}=1440 \mathrm{~kg} / \mathrm{m}^{3}$ for fiber scaffold). The elastic modulus of the fiber bundles in model II was set at $70 \mathrm{GPa}$. The finite element mesh contains a total of 2,137,387 elements. In these simulations, the target was set into a rigid ball with radius of $1 \times 10^{-6} \mathrm{~m}$ and mass of $1 \times 10^{-16} \mathrm{~kg}$. The contact between the model and target was defined as frictionless (tangential) and hard (normal). 
Scanning electron microscopy (SEM). Whole or polished dactyl clubs were examined with a scanning electron microscope (Hitachi S-4800) at an accelerating voltage of $10 \mathrm{keV}$. To prevent surface charging, the samples were coated with Pt.

\section{Data Availability}

All raw data that support the findings in this study are available from the corresponding authors upon request.

\section{References}

1. Obradovic, J., Boria, S. \& Belingardi, G. Lightweight design and crash analysis of composite frontal impact energy absorbing structures. Composite Structures 94, 423-430, doi:https://doi.org/10.1016/j.compstruct.2011.08.005 (2012).

2. Muneer Ahmed, M., Dhakal, H. N., Zhang, Z. Y., Barouni, A. \& Zahari, R. Enhancement of impact toughness and damage behaviour of natural fibre reinforced composites and their hybrids through novel improvement techniques: A critical review. Composite Structures 259, 113496, doi:https://doi.org/10.1016/j.compstruct.2020.113496 (2021).

3. Ritchie, R. O. The conflicts between strength and toughness. Nature Materials 10, 817-822, doi:10.1038/nmat3115 (2011).

4. Huang, W. et al. Multiscale Toughening Mechanisms in Biological Materials and Bioinspired Designs. Advanced Materials 31, doi:10.1002/adma.201901561 (2019).

5. Liu, Z. et al. Enhanced protective role in materials with gradient structural orientations: Lessons from Nature. Acta Biomaterialia 44, 31-40, doi:https://doi.org/10.1016/j.actbio.2016.08.005 (2016).

6. Naleway, S. E., Taylor, J. R. A., Porter, M. M., Meyers, M. A. \& McKittrick, J. Structure and mechanical properties of selected protective systems in marine organisms. Materials Science \& Engineering CMaterials for Biological Applications 59, 1143-1167, doi:10.1016/j.msec.2015.10.033 (2016).

7. Wegst, U. G. K., Bai, H., Saiz, E., Tomsia, A. P. \& Ritchie, R. O. Bioinspired structural materials. Nature Materials 14, 23-36, doi:10.1038/nmat4089 (2015).

8. Grunenfelder, L. K. et al. Bio-inspired impact-resistant composites. Acta Biomaterialia 10, 3997-4008, doi:10.1016/j.actbio.2014.03.022 (2014).

9. Zimmermann, E. A. et al. Mechanical adaptability of the Bouligand-type structure in natural dermal armour. Nature Communications 4, 7, doi:10.1038/ncomms3634 (2013).

10. Yao, H.-B., Ge, J., Mao, L.-B., Yan, Y.-X. \& Yu, S.-H. 25th Anniversary Article: Artificial Carbonate Nanocrystals and Layered Structural Nanocomposites Inspired by Nacre: Synthesis, Fabrication and Applications. 26, 163-188, doi:https://doi.org/10.1002/adma.201303470 (2014).

11. Launey, M. E., Chen, P. Y., McKittrick, J. \& Ritchie, R. O. Mechanistic aspects of the fracture toughness of elk antler bone. Acta Biomaterialia 6, 1505-1514, doi:https://doi.org/10.1016/j.actbio.2009.11.026 (2010). 
12. Patek, S. N., Korff, W. L. \& Caldwell, R. L. Deadly strike mechanism of a mantis shrimp. Nature 428, 819-820, doi:10.1038/428819a (2004).

13. Claverie, T., Chan, E. \& Patek, S. N. Modularity and scaling in fast movements: power amplification in mantis shrimp. Evolution 65, 443-461, doi:10.1111/j.1558-5646.2010.01133.x (2011).

14. Patek, S. N. \& Caldwell, R. L. Extreme impact and cavitation forces of a biological hammer: strike forces of the peacock mantis shrimp Odontodactylus scyllarus. Journal of Experimental Biology 208, 3655-3664, doi:10.1242/jeb.01831 (2005).

15. Weaver, J. C. et al. The Stomatopod Dactyl Club: A Formidable Damage-Tolerant Biological Hammer. Science 336, 1275-1280, doi:10.1126/science.1218764 (2012).

16. Amini, S. et al. Textured fluorapatite bonded to calcium sulphate strengthen stomatopod raptorial appendages. Nature Communications 5, doi:10.1038/ncomms4187 (2014).

17. Amini, S., Tadayon, M., Idapalapati, S. \& Miserez, A. The role of quasi-plasticity in the extreme contact damage tolerance of the stomatopod dactyl club. Nature Materials 14, 943-+, doi:10.1038/nmat4309 (2015).

18. Yaraghi, N. A. et al. A Sinusoidally Architected Helicoidal Biocomposite. Advanced Materials 28, 6835-+, doi:10.1002/adma.201600786 (2016).

19. Grunenfelder, L. K. et al. Ecologically Driven Ultrastructural and Hydrodynamic Designs in Stomatopod Cuticles. Advanced Materials 30, doi:10.1002/adma.201705295 (2018).

20. Huang, W. et al. A natural impact-resistant bicontinuous composite nanoparticle coating. Nature Materials 19, 1236-+, doi:10.1038/s41563-020-0768-7 (2020).

21. Chua, J. Q. I., Srinivasan, D. V., Idapalapati, S. \& Miserez, A. Fracture toughness of the stomatopod dactyl club is enhanced by plastic dissipation: A fracture micromechanics study. Acta Biomaterialia 126, 339-349, doi:https://doi.org/10.1016/j.actbio.2021.03.025 (2021).

22. Han, Q. et al. Study on mechanical properties of multi -structure dactyl -inspired sandwich honeycomb with basalt fiber. Composite Structures 247, doi:10.1016/j.compstruct.2020.112467 (2020).

23. Liu, P., Duan, H., Le Van, L., Ye, T. \& Tinh Quoc, B. Buckling of stomatopod-dactyl-club-inspired functional gradient plates: A numerical study. Composite Structures 207, 801-815, doi:10.1016/j.compstruct.2018.09.078 (2019).

24. Rivera, J., Yaraghi, N. A., Huang, W., Gray, D. \& Kisailus, D. Modulation of impact energy dissipation in biomimetic helicoidal composites. Journal of Materials Research and Technology 9, 14619-14629, doi:https://doi.org/10.1016/j.jmrt.2020.10.051 (2020).

25. Zhang, Y. et al. Novel X-Ray and Optical Diagnostics for Studying Energetic Materials: A Review. Engineering 6, 992-1005, doi:https://doi.org/10.1016/j.eng.2020.06.019 (2020).

26. Takeuchi, A. \& Suzuki, Y. Recent progress in synchrotron radiation 3D-4D nano-imaging based on Xray full-field microscopy. Microscopy 69, 259-279, doi:10.1093/jmicro/dfaa022 (2020). 
27. Nomura, K. et al. Interaction and coalescence of nanovoids and dynamic fracture in silica glass: multimillion-to-billion atom molecular dynamics simulations. J. Phys. D-Appl. Phys. 42, 12, doi:10.1088/0022-3727/42/21/214011 (2009).

28. Suksangpanya, N., Yaraghi, N. A., Kisailus, D. \& Zavattieri, P. Twisting cracks in Bouligand structures. Journal of the Mechanical Behavior of Biomedical Materials 76, 38-57, doi:10.1016/j.jmbbm.2017.06.010 (2017).

29. Suksangpanya, N., Yaraghi, N. A., Pipes, R. B., Kisailus, D. \& Zavattieri, P. Crack twisting and toughening strategies in Bouligand architectures. International Journal of Solids and Structures 150, 83-106, doi:10.1016/j.ijsolstr.2018.06.004 (2018).

30. Launey, M., Buehler, M. \& Ritchie, R. On the Mechanistic Origins of Toughness in Bone. Annual Review of Materials Research 40, 25-53, doi:10.1146/annurev-matsci-070909-104427 (2010).

31. Fantner, G. E. et al. Influence of the degradation of the organic matrix on the microscopic fracture behavior of trabecular bone. Bone 35, 1013-1022, doi:10.1016/j.bone.2004.05.027 (2004).

32. Bajaj, D. \& Arola, D. D. On the R-curve behavior of human tooth enamel. Biomaterials 30, 4037-4046, doi:https://doi.org/10.1016/j.biomaterials.2009.04.017 (2009).

33. Chen, P.-Y., Lin, A. Y.-M., McKittrick, J. \& Meyers, M. A. Structure and mechanical properties of crab exoskeletons. Acta Biomaterialia 4, 587-596, doi:https://doi.org/10.1016/j.actbio.2007.12.010 (2008).

34. Zhang, Y. et al. Molecular to Macroscale Energy Absorption Mechanisms in Biological Body Armour Illuminated by Scanning X-ray Diffraction with In Situ Compression. Acs Nano 14, 16535-16546, doi:10.1021/acsnano.0c02879 (2020).

35. Tiu, J., Belli, R. \& Lohbauer, U. Thickness influence of veneering composites on fiber-reinforced systems. Dental Materials 37, 477-485, doi:https://doi.org/10.1016/j.dental.2020.12.002 (2021).

36. Amini, S. et al. A diecast mineralization process forms the tough mantis shrimp dactyl club. Proceedings of the National Academy of Sciences of the United States of America 116, 8685-8692, doi:10.1073/pnas.1816835116 (2019).

37. Fratzl, P., Gupta, H., Fischer, F. \& Kolednik, O. Hindered Crack Propagation in Materials with Periodically Varying Young's Modulus-Lessons from Biological Materials. Advanced Materials 19, 2657-2661, doi:10.1002/adma.200602394 (2007).

38. Cook, J., Evans, C. C., Gordon, J. E. \& Marsh, D. M. MECHANISM FOR CONTROL OF CRACK PROPAGATION IN ALL-BRITTLE SYSTEMS. Proc. R. Soc. Lond. A-Math. Phys. Sci. 282, 508-+, doi:10.1098/rspa.1964.0248 (1964).

39. Cao, Y., Wang, W., Wang, J. \& Zhang, C. Experimental and numerical study on tensile failure behavior of bionic suture joints. Journal of the Mechanical Behavior of Biomedical Materials 92, 40-49, doi:https://doi.org/10.1016/j.jmbbm.2019.01.001 (2019).

40. Bai, H., Polini, A., Delattre, B. \& Tomsia, A. P. Thermoresponsive Composite Hydrogels with Aligned Macroporous Structure by Ice-Templated Assembly. Chem. Mat. 25, 4551-4556, doi:10.1021/cm4025827 (2013). 
41. Lin, E., Li, Y., Ortiz, C. \& Boyce, M. C. 3D printed, bio-inspired prototypes and analytical models for structured suture interfaces with geometrically-tuned deformation and failure behavior. Journal of the Mechanics and Physics of Solids 73, 166-182, doi:https://doi.org/10.1016/j.jmps.2014.08.011 (2014).

42. Chen, R.-C. et al. PITRE: software for phase-sensitive X-ray image processing and tomography reconstruction. Journal of Synchrotron Radiation 19, 836-845, doi:10.1107/S0909049512029731 (2012).

\section{Declarations}

\section{Acknowledgments}

This work was supported by National Key R\&D Program of China (2018YFC0309800), National Basic Research Program of China (2017YFA0504900), One-hundred Talents Program (Y851552) of Chinese Academy of Sciences and Strategic Priority Research Program of Chinese Academy of Sciences (XDB 37000000), the National Science Foundation for Young Scientists of China (Grant No. 12005253) and NSFC『Grant No. 11627901). HSG thanks the BBSRC (BB/R003610/1), MRC (MR/R025673/1) and EPSRC (EP/V011235/1) for research grant funding. All authors gratefully acknowledge the support from the 4W1A and 3W1 beamlines of Beijing Synchrotron Radiation Facility (BSRF) for nano-CT and 2D fast x-ray imaging measurements and BL13W1 beamline of Shanghai Synchrotron Radiation Facility (SSRF) for micro-CT measurement.

\section{Author contributions}

Y.Z. initiated and supervised the whole project. Z.D. performed all microscopy observations, performed and analyzed micro- and nano-CT characterization and performed DFEA. Z.D., S. C., Y.M.Y. and Y.Y.Z performed in situ characterization. X.Y.Z and G.C.C helped perform nano-CT characterization. J.X. helped with the DFEA. H.S.G., Y.H.D. and S.N.L. helped the with project design and refined the manuscript. The manuscript was written through contributions of Z.D. and Y.Z. All authors have given approval to the final version of the manuscript.

\section{Competing interests}

The authors declare no competing interests.

\section{Figures}



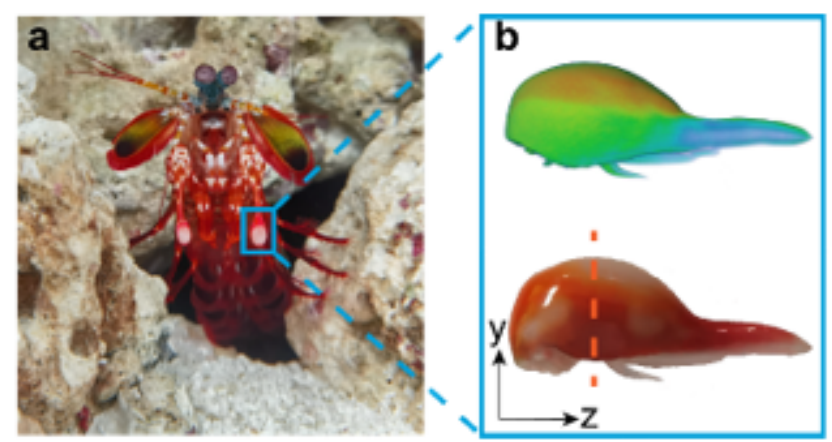

\section{d}
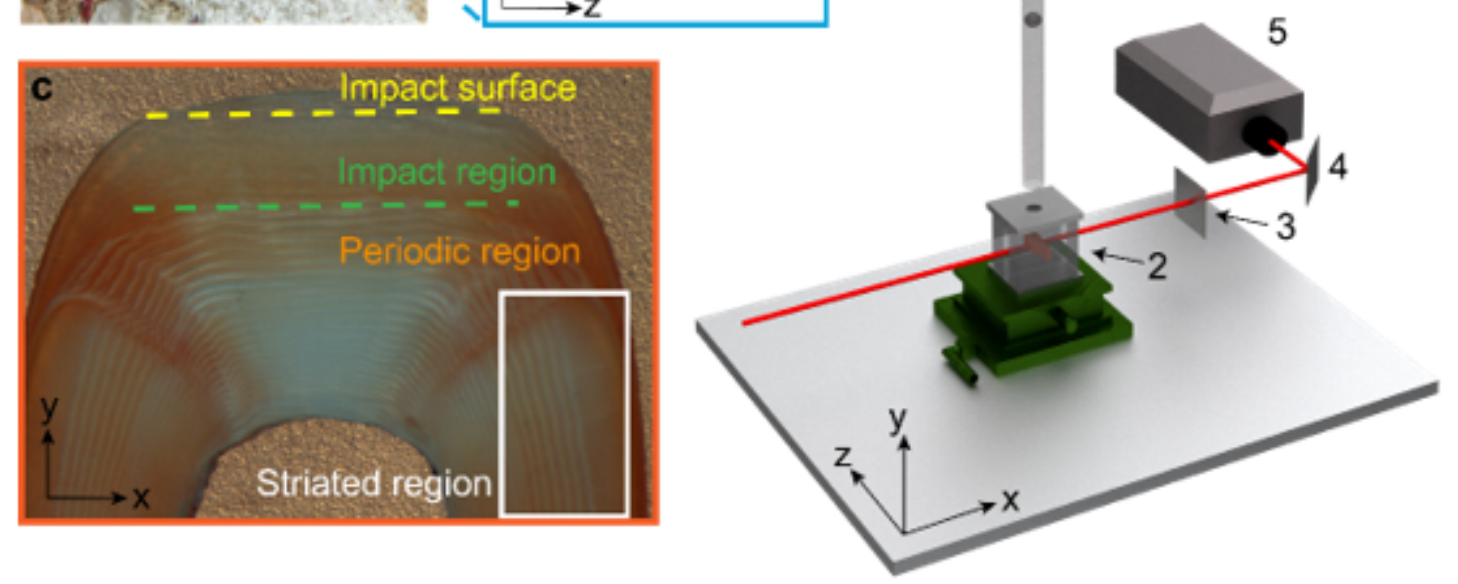

Figure 1

Samples and experimental setup. (a): Photography of Odontodactylus scyllarus with dactyl club indicated with blue square. (b): Top: 3D rendering of dactyl club based on lab CT result. Bottom: Photography of the dactyl club separated from raptorial appendage. (c): Optical microscopy image of a cross section of dactyl club as denoted in b (sectioned along the red dashed line). The impact surface, impact region, periodic region and striated region are depicted in c. (d): Schematic shows the experiment setup for the in situ impact test equipped a high-speed $x$-ray imaging system. 1: gas gun; 2: sample holder; 3: scintillator;4: mirror ,5: high-speed camera. 

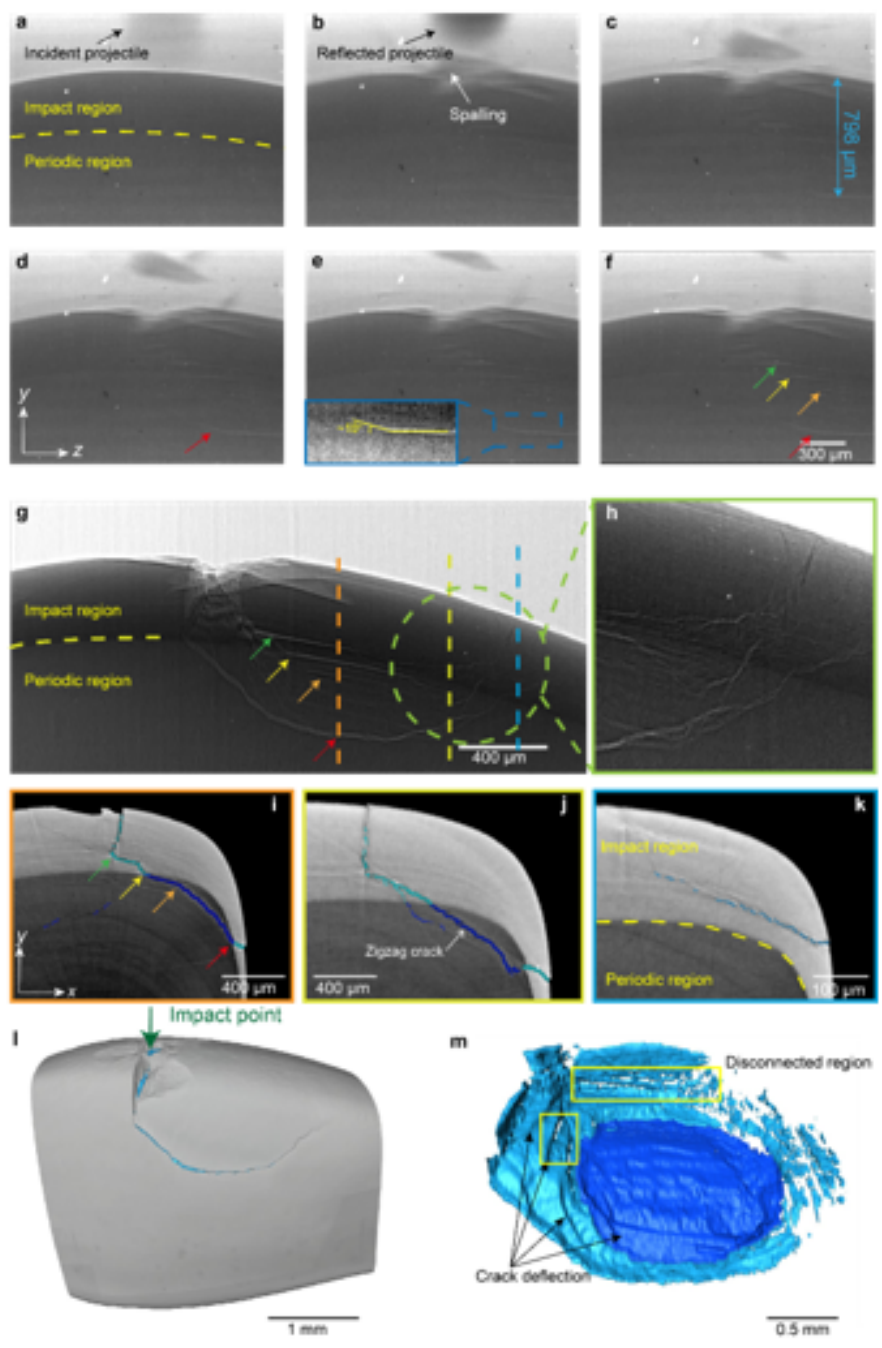

Figure 2

Dynamic structure deformation and crack evolution observed via in situ 2D fast imaging and high resolution 3D postmortem CT examination. (a-f): Snapshots of dactyl club under dynamic impact with time intervals of $6 \mu \mathrm{s}$. Catastrophic spalling and clear crack deflections can be spotted in situ. Image e shows the propagating crack started to deflect towards (highlighted in yellow line) the impacting point at $33 \mu \mathrm{s}$ after the exact impacting moment, which grew further (indicated by red arrow in $\mathrm{g}$ ) and merged with another crack system nucleated right under the impacting point as shown in $\mathrm{g}$. Image g displays a projection of the impacted dactyl club from an ex-situ CT test at a similar perspective as in the in situ 2D imaging, with some major deflection sites indicated with arrows. Image h shows crack branching behaviors while they were deflected towards the surface of dactyl club. (i-k): Slices of CT reconstruction of damaged dactyl club denoted in $\mathrm{g}$. The main crack was deflected around the interface of impactperiodic region. Within periodic region, the crack was deflected periodically forming a zigzag-shaped crack path. At the end of main crack, the crack branched into microcracks and propagated to the surface of dactyl club as shown in $\mathrm{k}$. (I): The 3D rendering of damaged dactyl club. (m): The 3D distribution of main crack system developed in dry dactyl club sample. (Dark bule: cracks within periodic region. Light blue: cracks within impact region.) 

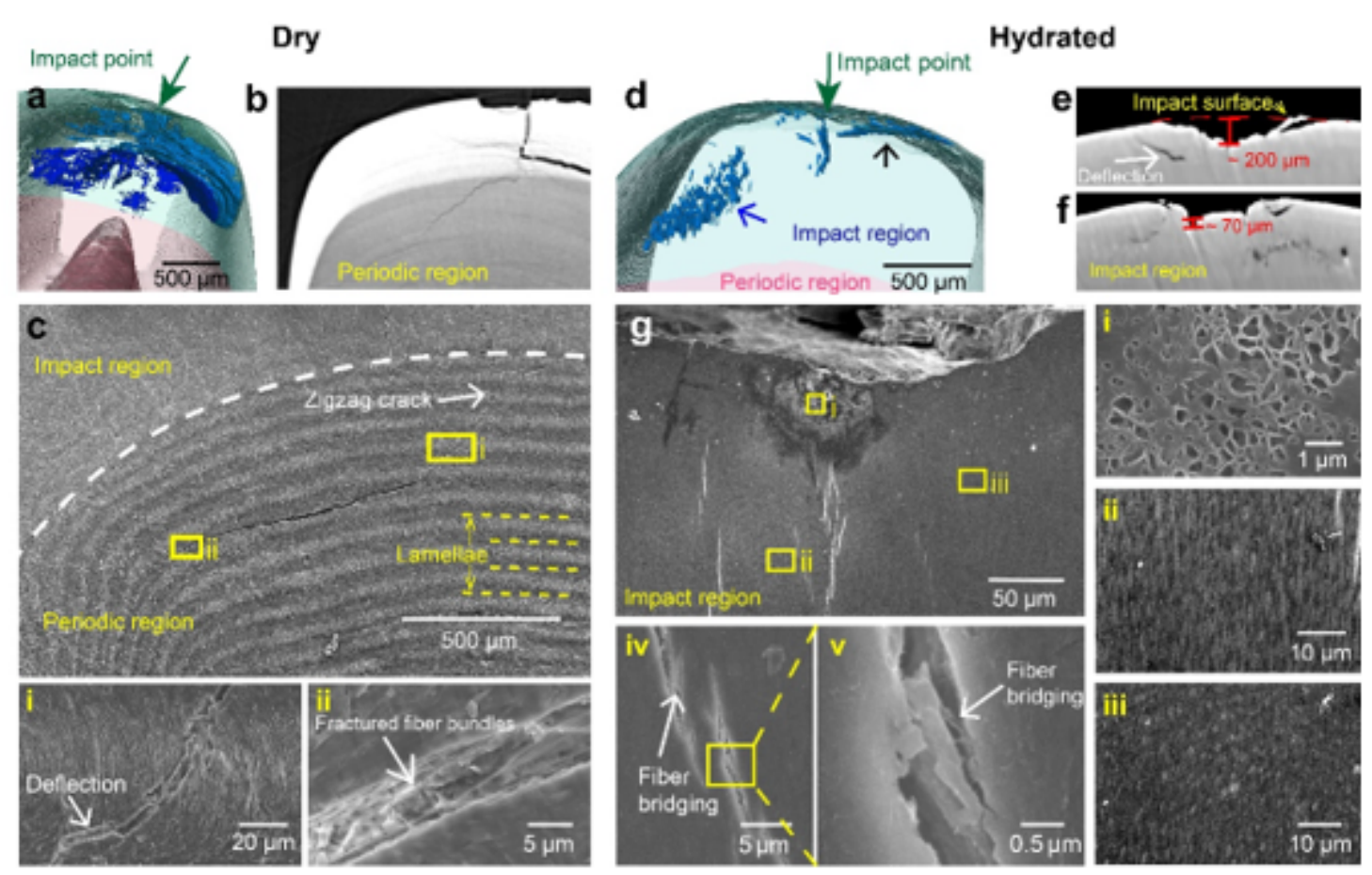

\section{Figure 3}

Influence of hydration state on the structural response of dactyl club subjected to impact. (a): The 3D distribution of main crack systems within dry dactyl club. A major crack system was formed under the loading point and propagated into the periodic region, which can be easily spotted from the representative slice (b) of the 3D reconstruction results. A zigzag-shaped crack path can be identified in the periodic region showing a characteristic crack deflection phenomenon found in this region. High resolution image of the zigzag-shaped crack path was successfully captured in SEM and shown in Supplementary Fig. 2a. (c): A SEM image shows the crack propagation within the periodic region, with image c-i shows crack penetrated through a lamella and c-ii shows the crack stopped after long distance propagation along the interface two neighboring lamellae. High magnification SEM image show fracture of fiber bundles at the crack stop site (c-ii and Supplementary Fig. 2b). (d): The 3D distribution of main crack systems within hydrated dactyl club. Differently, no major cracks developed right under the loading point but several separate cracks formed surrounding the loading point. The main cracks propagated to the interface of the impact surface and impact region and then get significantly deflected, which can also be easily spotted from representative slices (e-f) of 3D reconstruction. (g): Low-magnification SEM image shows both plastic and elastic regions formed beneath the impact point. (g-i): A region featuring with large pores due to the loss of mineral contents. (g-ii) Enlarged view shows a typical plastic zone featuring with highly misaligned mineral crystallites. (g-iii) Enlarged view shows an elastic zone featuring with textured arrangement of mineral crystallites. g-iv and g-v show the fiber bridging behind crack tips and between fractured fiber bundles within the plastic zone. 

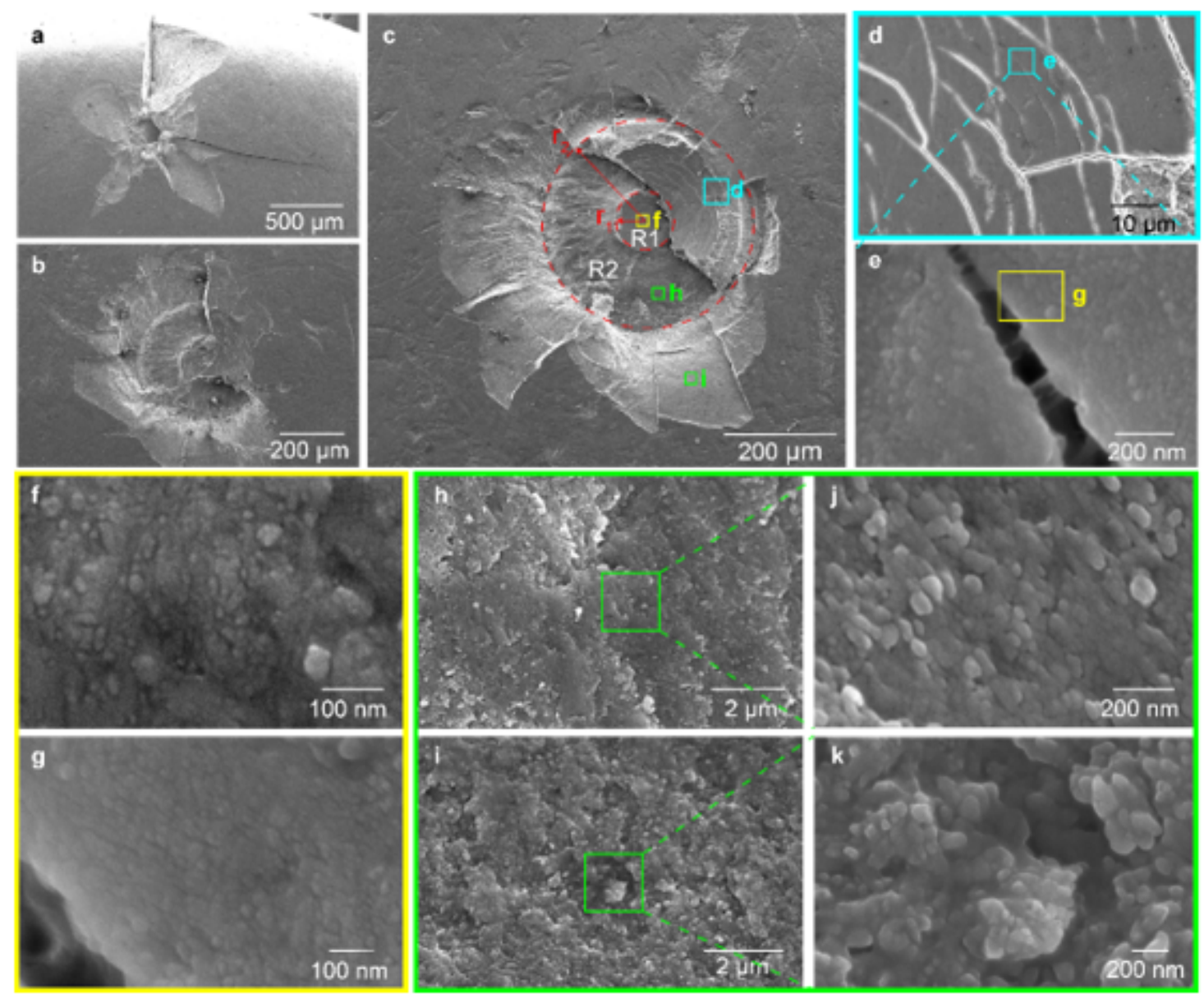

Figure 4

Different morphologies and deformation mechanisms in the damaged dactyl surface in varying hydration states. (a): SEM image of the damaged surface of dry dactyl club sample (impacted with speed of 160 $\mathrm{m} / \mathrm{s}$ ); (b-c): SEM images of the damaged surface of hydrated dactyl club samples, impacted with speed of $160 \mathrm{~m} / \mathrm{s}$ and $200 \mathrm{~m} / \mathrm{s}$, respectively. For convenience, we labeled the surface area with R1 and R2 region according to different radius from the central impacting point. (d): Plenty of circular microcracks were found on the R2 region of wet dactyl sample. (e): High-magnification SEM image shows large amounts of fiber bridges existing at the crack tip. $(\mathrm{f})$ : The mineral particles were break into secondary particles at the impacted point, while they were kept relatively intact at sites away from impacted point (g). (h-i): Low-magnification SEM images of fiber layers collected from regions R2 (h) and the outer region (i). (j-k): High-magnification SEM images show the morphology states of the mineral nanoparticle in h-i. 

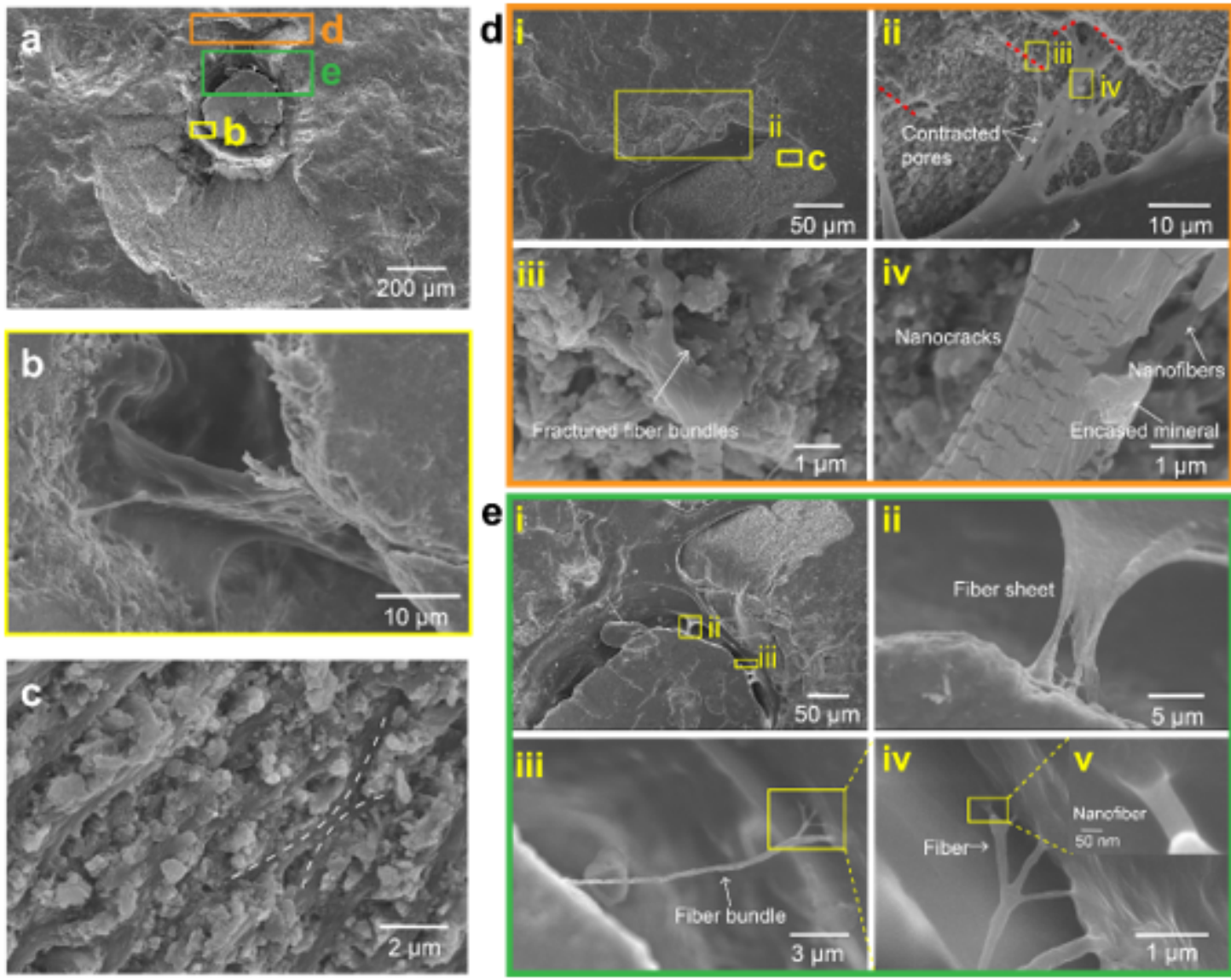

\section{Figure 5}

Multiscale fiber bridging within the impacted dactyl club. (a): SEM image of damaged surface of dactyl clubs. (b): A twisted fiber bridge formed within a large crack which links multiple fiber layers in the helicoidal architected structure. (c): The SEM image of intersecting fibers layers marked in $d$. (d): $d-i$ shows the fiber bridging away from impact point. $d$-ii shows the in-plane fiber were pulling put from different layers (highlight with red lines). d-iii shows the out-of-plane fiber bundles fractured while the inplane fiber bundles were pulling out. (d-iv): The nanocracks in encased mineral of nanofibers and the separation of clustered nanofibrils. (e): Fiber bridging near the impact point, illustrating the hierarchical fiber bridging: fractured fiber sheet (e-ii), fiber bundle (e-iii), fiber (e-iv) and nanofiber (e-v). 

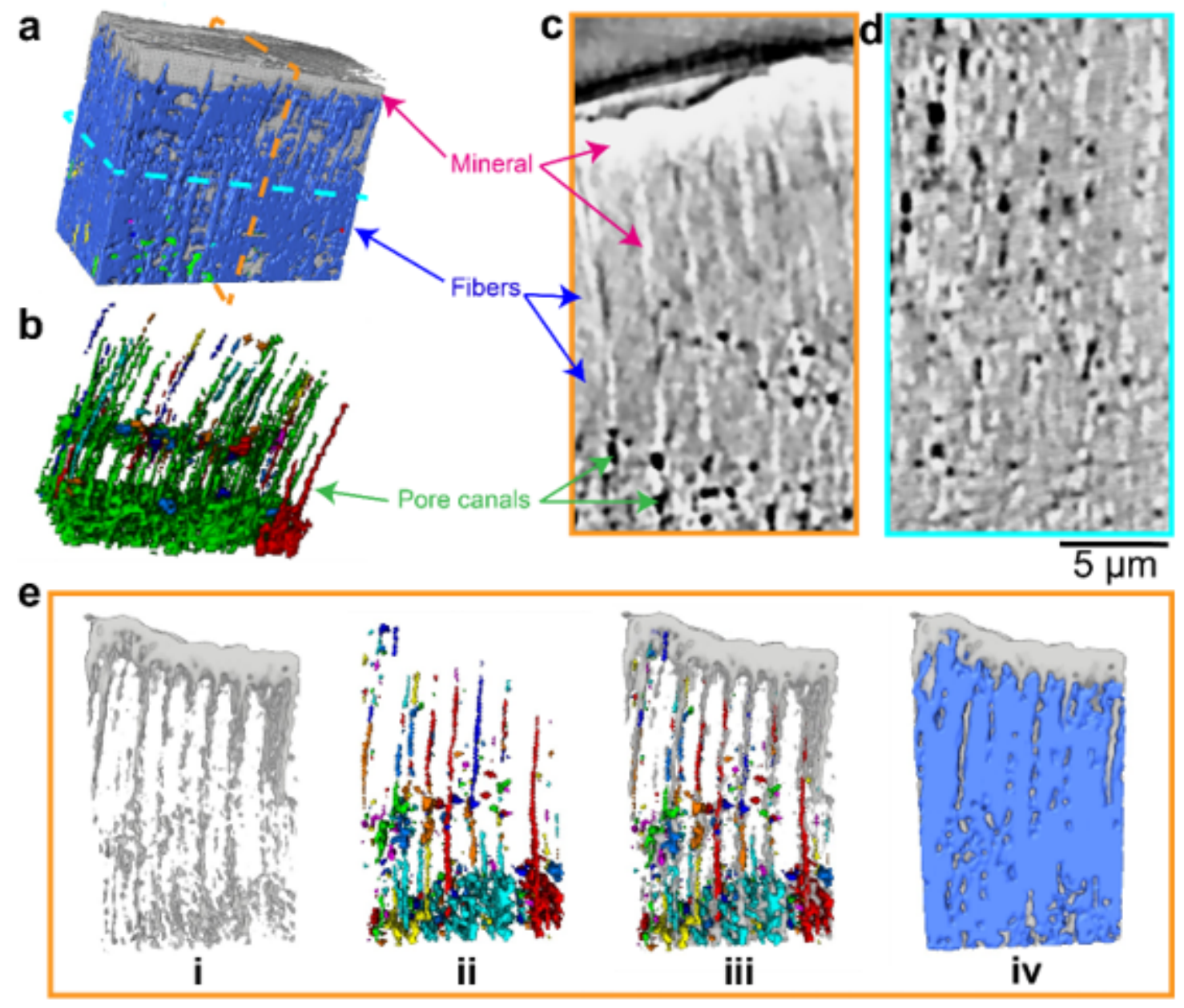

\section{Figure 6}

3D architecture of the impact surface and impact region revealed by nano-CT characterization. (a): A highly interpenetrated 3D structure consisting by three main segments: mineral nanoparticle aggregation, chitin fiber scaffold and pore canal network. (b): 3D model of pore canal network. (c-d): Slices of CT scan acquired from perpendicular projections (White regions represent mineral particles, grey regions represent chitin fiber bundles and black regions represent pore canals.). (e): The 3D rendering of interest volume. The distributions of pore canals (e-ii) and minerals are highly correlated (e-iii) shown in colored CT slices, with e-i showing a rep of the mineral particle distribution. While (e-iv) shows the interlocking structural design of mineral particles and fiber bundles in the impact surface and impact region. 

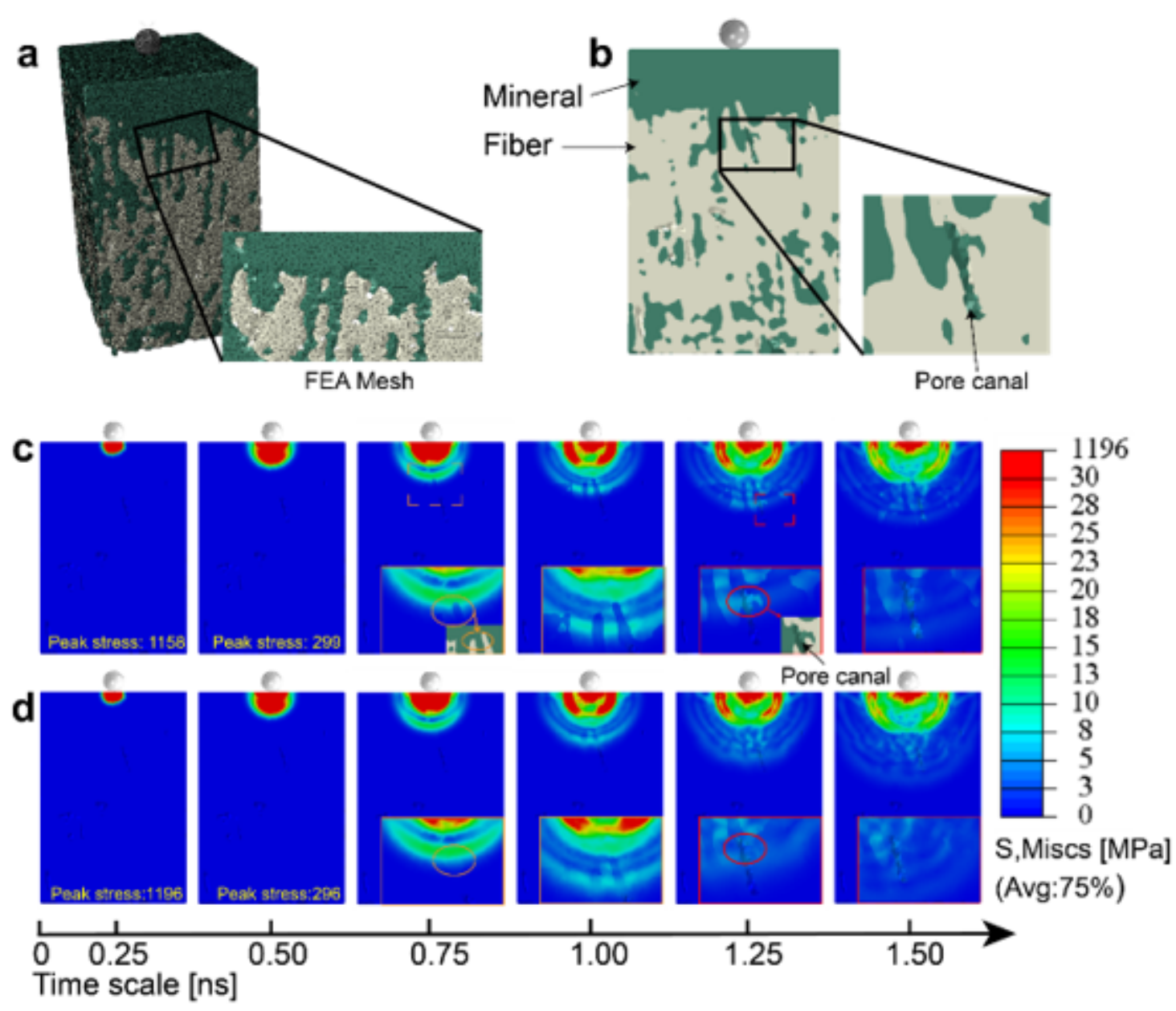

Figure 7

DFEM simulation for structure and mechanical analysis of impact surface and impact region. (a): The 3D schematic of our model following the complex microscale geometry of impact surface and impact region. Color-coding corresponds to the different components. As shown in $b$ the interlocking structural design of mineral particles and fiber bundles as well as the distribution mineral coated of pore canals were fidelity retained in our model. $c$ and $d$ correspond to average Von-mises stress distribution cloud diagrams of models with mismatch (c) and uniform (d) elastic modulus, respectively. The peak Vonmises stress were shown at the bottom right corner of certain figures.

\section{Supplementary Files}

This is a list of supplementary files associated with this preprint. Click to download.

- supplementary.docx 